\title{
Uso de plantas medicinais e fitoterápicos no climatério e menopausa
}

\author{
Use of medicinal and phytotherapy plants in climate and menopause \\ Uso de plantas medicinales y herbales em clima y menopausia
}

Recebido: 26/07/2021 | Revisado: 30/07/2021 | Aceito: 03/08/2021 | Publicado: 08/08/2021

\author{
Ana Katarina Dias de Oliveira \\ ORCID: https://orcid.org/0000-0003-4609-5992 \\ Universidade do Estado do Rio Grande do Norte, Brasil \\ E-mail: akatarinadoli@gmail.com \\ Kalyane Kelly Duarte de Oliveira \\ ORCID: https://orcid.org/0000-0001-7713-3264 \\ Universidade Federal do Rio Grande do Norte, Brasil \\ Email: kenfoliveira@gmail.com \\ Lara Barbosa de Souza \\ ORCID: https://orcid.org/0000-0003-4730-1401 \\ Universidade Potiguar, Brasil \\ Email: larabiotec@gmail.com \\ Rejane Helena Pereira Lins \\ ORCID: https://orcid.org/0000-0001-9479-6174 \\ Universidade Federal Rural do Semi-Árido, Brasil \\ Email: rejanehelena@gmail.com
}

\begin{abstract}
Resumo
Objetivo: investigar o uso de plantas medicinais no tratamento dos sintomas do climatério, identificando quais as plantas medicinais mais usadas e para quais sintomatologias, investigar a relação entre o uso popular e a recomendação científica dessas plantas. Metodologia: estudo descritivo, com abordagem quantitativa, com mulheres entre 45 a 59 anos que apresentavam alguma sintomatologia de climatério, não tiveram menopausa precoce nem por indução de procedimentos cirúrgicos. Resultados: do total de 85 participantes, 95,3\% ( $\mathrm{n}=81)$ apresentam ondas de calor, seguido de irritabilidade, ressecamento vaginal e insônia que correspondeu a 57,6\% ( $n=49)$ 49,4\% ( $n=42)$ e 49,4\% ( $\mathrm{n}=42)$ respectivamente. $41,1 \%(\mathrm{n}=35)$ participantes usam algum tipo de plantas ou fitoterápicos de forma combinada ou não para tratar os sintomas. $18,8 \%(\mathrm{n}=16)$ usam medicamentos alopáticos ou hormonais e $58,9 \%$ $(n=28)$ não usam nada. As plantas indicadas para insônia e ansiedade, como a camomila, capim santo e cidreira foram as mais mencionadas pelas participantes, algumas como a canela citada na pesquisa como tranquilizante não tem respaldo científico para essa finalidade. Todos os fitoterápicos mencionados tinham respaldado científico. A prescrição de fitoterápicos por profissionais de saúde aconteceu principalmente nos atendimentos realizados no ambulatório da faculdade de medicina. Considerações finais: apesar de muitas mulheres apresentarem sintomas do climatério, percebe-se que poucas têm conhecimento sobre fitoterápicos e plantas medicinais para essa finalidade, a maioria usa plantas na forma de chá para insônia e ansiedade sem saber a relação desses sintomas com o climatério e nem todas as substâncias utilizadas tem respaldo científico.
\end{abstract}

Palavras-chave: Climatério; Fitoterapia; Menopausa; Plantas medicinais.

\begin{abstract}
Objective: to investigate the use of medicinal plants in the treatment of climacteric symptoms, identifying which medicinal plants are most used and for which symptoms, to investigate the relationship between popular use and scientific recommendation of these plants. Methodology: descriptive study, with a quantitative approach, with women aged 45 to 59 years who presented some symptoms of menopause, did not experience early menopause or due to induction of surgical procedures. Results: of the total of 85 participants, $95.3 \%(\mathrm{n}=81)$ had hot flashes, followed by irritability, vaginal dryness and insomnia which corresponded to $57.6 \%(n=49) 49.4 \%(n=42)$ and $49.4 \%(n=42)$ respectively. $41.1 \%(n=35)$ participants use some type of plant or herbal medicine in combination or not to treat symptoms. $18.8 \%(\mathrm{n}=16)$ use allopathic or hormonal drugs and $58.9 \%(\mathrm{n}=28)$ do not use anything. Plants indicated for insomnia and anxiety, such as chamomile, capim santo and lemon balm were the most mentioned by the participants, some such as cinnamon mentioned in the research as a tranquilizer have no scientific support for this purpose. All mentioned herbal medicines had scientific backing. The prescription of phytotherapeutic drugs by health professionals took place mainly in the consultations carried out at the outpatient clinic of the Faculty of Medicine. Final considerations: although many women have symptoms of the climacteric, it is noticed that few have knowledge about herbal medicines and medicinal plants for this purpose, most use plants in the form of tea for insomnia and anxiety without knowing the relationship of these symptoms with the climacteric and not all substances used have scientific backing.
\end{abstract}

Keywords: Climacteric; Phytotherapy; Menopause; Medicinal plants. 


\begin{abstract}
Resumen
Objetivo: investigar el uso de plantas medicinales en el tratamiento de síntomas climatéricos, identificando qué plantas medicinales son más utilizadas y para qué síntomas, investigar la relación entre el uso popular y la recomendación científica de estas plantas. Metodología: estudio descriptivo, con abordaje cuantitativo, con mujeres de 45 a 59 años que presentaban algún síntoma de menopausia, no experimentaron menopausia precoz o por inducción de procedimientos quirúrgicos. Resultados: del total de 85 participantes, el 95,3\% $(\mathrm{n}=81)$ presentó sofocos, seguido de irritabilidad, sequedad vaginal e insomnio que correspondieron al $57,6 \%(\mathrm{n}=49) 49,4 \%(\mathrm{n}=42)$ y $49,4 \%(n=42)$ respectivamente. El 41,1\% $(\mathrm{n}=35)$ de los participantes usa algún tipo de medicina vegetal o herbal en combinación o no para tratar los síntomas. El 18,8\% $(\mathrm{n}=16)$ utiliza fármacos alopáticos u hormonales y el 58,9\% $(\mathrm{n}=28)$ no utiliza nada. Plantas indicadas para el insomnio y la ansiedad, como la manzanilla, el capim santo y el toronjil fueron las más mencionadas por los participantes, algunas como la canela mencionada en la investigación como tranquilizante no tienen sustento científico para tal fin. Todas las medicinas a base de hierbas mencionadas tenían respaldo científico. La prescripción de fitoterápicos por parte de los profesionales de la salud se realizó principalmente en las consultas realizadas en el ambulatorio de la facultad de medicina. Consideraciones finales: si bien muchas mujeres presentan síntomas del climaterio, se advierte que pocas tienen conocimiento sobre las plantas medicinales y plantas medicinales para tal fin, la mayoría usa plantas en forma de té para el insomnio y la ansiedad sin conocer la relación de estos síntomas con la climatérico y no todas las sustancias utilizadas tienen respaldo científico.

Palabras clave: Climaterio; Fitoterapia; Menopausia; Plantas medicinales.
\end{abstract}

\title{
1. Introdução
}

O climatério é um período fisiológico caracterizado por alterações hormonais, principalmente redução na produção de estrógeno decorrente da diminuição da função ovariana que marca a transição entre a fase reprodutiva para a fase não reprodutiva da mulher. Geralmente inicia-se por volta da quarta década de vida da mulher e pode se estender até os 65 anos, embora ocorra com mais frequência entre os 45 aos 59 anos e tem seu ápice com a menopausa definida como último ciclo menstrual confirmado após 12 meses ininterruptos de ausência de menstruação, sem outras causas patológicas, psicológicas ou induzidas, como cirurgias de histerectomia (Brasil, 2008a).

Embora muitas vezes seja tratado como uma endocrinopatia por causa dos sintomas e desconfortos provocados em algumas mulheres (Brasil, 2008a; Cardoso \& Camargo, 2017), é considerado pela Organização Mundial de Saúde (OMS) uma fase biológica natural da mulher e não um processo patológico. Estudos mostram que $60 \%$ a $80 \%$ das mulheres que vivenciam essa fase apresentam sintomas climatéricos, e isso pode ter implicações significativas na qualidade de vida (Fernandes \& Farias, 2017; Silva \& Mamede, 2020).

Com o envelhecimento da população brasileira, o aumento da expectativa de vida das mulheres que atualmente está em torno de 72 anos no país, e o fenômeno da feminização da velhice credita-se que um grande número de mulheres irá conviver com sinais e sintomas decorrentes do climatério por vários anos de vida (Xavier \&Trindade, 2018).

Os sintomas característicos do climatério podem envolver problemas vasomotores, urogenitais, sexuais, psíquicos e distúrbios do sono. Sendo as ondas de calor, disfunções sexuais, atrofia e ressecamento vaginal, alterações no perfil lipídico e distúrbios psíquicos os sintomas mais comuns entre as mulheres. Algumas mulheres passam por essa fase da vida sem sintomas, enquanto outras podem apresentar sintomatologias diversas e com intensidades variadas (Albuquerque et al., 2019; Manica, Bellaver \& Zancanaro, 2019) .

Há algumas décadas a terapia de reposição hormonal vem sendo a prática mais comum na medicina para tratamento dos sintomas do climatério, sendo considerada por alguns profissionais de saúde um dos tratamentos mais eficazes (Wender, Pompei \& Fernandes, 2014). Porém, a terapia hormonal pode provocar efeitos colaterais como aumento dos riscos de neoplasias mamárias, embolias pulmonares e demências, além de sangramentos irregulares, edemas, mastalgias, náuseas, câimbras, irritabilidade, cefaleia e ganho de peso (Aidelsburger, 2012; Franciscis et al., 2019).

As possíveis complicações que podem ocorrer durante a terapia de reposição hormonal, bem como, receio dos efeitos colaterais, as contraindicações, dificuldades de acesso e o custo financeiro, favorecem a procura por terapias complementares não medicamentosas entre elas as plantas medicinais e fitoterápicos (Barra et al., 2014; Franciscis et al., 2019). A Política 
Nacional de Atenção Integral à Saúde da Mulher no tópico sobre saúde da mulher no climatério e menopausa aborda a necessidade de conscientização dos direitos e das possibilidades de terapêuticas para essa fase da vida como uma forma de enfrentamento da medicalização, principalmente a prescrição hormonal, que ainda é um tema controverso na literatura.

Em 2006 da Política Nacional de Plantas Medicinais e Fitoterápicos trouxe respaldo legal as práticas integrativas no Brasil. Em 2008 o Programa Nacional de Plantas Medicinais e Fitoterápicos é implantado, e entre seus princípios norteadores pode-se destacar: Ampliação das opções terapêuticas e melhoria da atenção à saúde aos usuários do Sistema Único de Saúde - SUS; Uso sustentável da biodiversidade brasileira; Valorização e preservação do conhecimento tradicional das comunidades e povos tradicionais (Brasil, 2009; Ibiapina et al., 2014).

Segundo Bueno et al., (2016), as plantas medicinais e fitoterápicos são importantes fontes de substâncias chamadas xenobióticas que visam uma melhoria das condições de saúde dos indivíduos que buscam tratamento. Estas substâncias tanto trazem benefícios como podem trazer algum desconforto, toxicidade ou interação com outros medicamentos, quando utilizadas sem orientação, sendo necessário o acompanhamento de um profissional (Franciscis et al., 2019).

Acredita-se que quando utilizadas corretamente, as plantas medicinais podem representar uma estratégia eficaz de baixo custo e menor efeito colateral para tratamento dos sintomas do climatério. Assim, estudos e pesquisas que abordem o uso de plantas medicinais constituem uma importante ferramenta para profissionais e usuárias que buscam tratamento para os sintomas do climatério fora da reposição hormonal. De acordo com a literatura entre as plantas medicinais e fitoterápicos mais utilizados para amenizar os sintomas do climatério estão: Camomila (Matricaria chamomilla), a Erva cidreira (Melissa officinalis), Black Cohosh (CimicifugaActea Racemosa L), Videira da Punctura (Tribulus Terrestres), Prímula (Oenotherabiennis), Alfafa (Medicago sativa),Valeriana Officinalis,Trevo Vermelho (Trifoliumpratense), Amora (Morus Alba L.) Gingkobiloba, isoflavona (Brasil, 2018; Rocha, Pereira \& Carneiro, 2018; Franciscis et al., 2019).

Nesse contexto esse trabalho tem como objetivo investigar o uso de plantas medicinais e fitoterápicos no tratamento de sintomas do climatério e menopausa. Buscando identificar quais as plantas medicinais mais usadas por mulheres e para quais sintomatologias e avaliar se essa utilização, caso exista, corresponde ao que é preconizado na literatura científica sobre as plantas medicinais por elas utilizadas.

\section{Metodologia}

Trata-se de uma pesquisa de caráter descritivo, com abordagem quantitativa. Para Sampieri; Collado e Lúcio (2013) os estudos descritivos tem por finalidade uma descrição detalhada dos variados aspectos que possibilitam a ocorrência de um determinado fenômeno.

A escolha pela abordagem quantitativa se deu por entender que esse tipo de estudo é previsível e estruturado. Permitindo que os resultados encontrados numa amostra possam ser generalizados para uma população maior. Além de estabelecer padrões de comportamentos de uma população (Sampieri; Collado; Lucio, 2013; Pereira et al, 2018).

A população estudada foi composta por mulheres na faixa etária de 45 a 59 anos de idade, residentes na área de adstrita da Unidade Básica de Saúde da zona urbana de Mossoró e, pelas participantes do grupo de atendimento à mulheres no climatério da Faculdade de Ciências da Saúde (FACS) da Universidade Regional do Rio Grande do Norte (UERN).

A amostra foi definida a partir do cálculo de populações finitas, com nível de confiança de 95\% e erro amostral de 5\%. Com base nesse cálculo o número de participantes da amostra foi definido em 158 mulheres, a partir de um universo de 266 mulheres. Entretanto quando aplicados os critérios de exclusão e respeitado o direito de cada mulher abordada aceitar participar voluntariamente da pesquisa, a amostra final contou com a participação de 85 mulheres que se adequaram aos critérios de inclusão e aceitaram participar, assinando o Termo de Consentimento Livre e Esclarecido (TCLE). 
Os critérios de inclusão foram: apresentarem sintomas característicos do climatério, estar na faixa etária entre 45 e 59 anos, morarem na área adstrita da referida unidade ou serem atendidas no ambulatório da FACS.

Foram excluídas da pesquisa mulheres que embora dentro da faixa etária dos 45 aos 59 anos relataram não apresentar sintomas do climatério, apresentaram sintomas de maneira precoce, antes dos 40 anos, ou induzido por métodos cirúrgicos como ooforectomia uni ou bilateral e histerectomia, que apresentavam problemas de fala que comprometesse a compreensão das respostas e distúrbio mentais.

O instrumento de coleta utilizado foi um questionário estruturado com perguntas objetivas. A coleta de dados aconteceu entre setembro de 2019 a fevereiro de 2020, após aprovação do Comitê de Ética e Pesquisa (CEP) da Universidade Estadual do Rio Grande do Norte (UERN) sob o número 3.302.296.

Os dados foram analisados pelo programa estatístico Statistical Package for the Social Sciences (SPSS) versão 23.0 sendo expressos em valores de média e desvio padrão, bem como mínimos, máximos, frequência simples e porcentagem. Sempre quando necessário, diferenças estatísticas entre as proporções de Sim e Não dos eventos relacionados ao uso de plantas medicinais no tratamento dos sintomas do climatério e menopausa foram obtidas através do teste binomial adotando-se uma proporção esperada de 0,5 . Valores de $\mathrm{p}<0,05$ foram considerados significativos.

\section{Resultados}

A amostra avaliada foi constituída por 85 mulheres com idades entre 45 anos (idade mínima) e 59 anos (idade máxima), sendo 68,2\% (n=58) acima de 50 anos, com prevalência de faixa etária entre 50 a 52, com média de 53,0 entre as participantes, e desvio padrão de 4,0 com apenas 31,8\% (n=27) abaixo dos 50 anos. Em relação ao nível de escolaridade houve uma predominância de níveis de instrução mais baixos, uma vez que 4,7\% (n=4) e 52,9 (n=45) dos participantes, informaram serem analfabetas e ter cursado até o $1^{\circ}$ grau, seja incompleto ou completo, respectivamente. Embora 3,5\% (n=3) participantes tenham concluído graduação, nenhuma ultrapassou esse nível de formação acadêmica.

A renda familiar da maioria das participantes era baixa, o que pode estar relacionado ao baixo nível de escolaridade das mesmas, como também pode influenciar o acesso a informação e aos serviços de saúde. A caracterização das participantes em relação a idade, escolaridade, renda e estado civil está descrita na Tabela 1. 
Tabela 1 - Valores de frequência simples e porcentagem da caracterização dos participantes $(\mathrm{n}=85)$.

\begin{tabular}{|c|c|c|}
\hline Item & Freq. & $\%$ \\
\hline Idade (anos) & & \\
\hline 41 a 50 & 27 & 31,8 \\
\hline Acima de 50 & 58 & 68,2 \\
\hline Média \pm desvio padrão & \multicolumn{2}{|c|}{$53,0 \pm 4,0$} \\
\hline Mínimo - máximo & \multicolumn{2}{|c|}{$45-59$} \\
\hline \multicolumn{3}{|l|}{ Escolaridade } \\
\hline Analfabeto & 04 & 4,7 \\
\hline Fundamental & 45 & 52,9 \\
\hline Médio & 33 & 38,8 \\
\hline Superior & 03 & 3,5 \\
\hline \multicolumn{3}{|l|}{ Renda } \\
\hline 1,00 & 40 & 47,1 \\
\hline 2,00 & 31 & 36,5 \\
\hline 3,00 & 11 & 12,9 \\
\hline 4,00 & 01 & 1,2 \\
\hline 5,00 & 02 & 2,4 \\
\hline \multicolumn{3}{|l|}{ Estado civil } \\
\hline Solteiro & 09 & 10,6 \\
\hline União estável & 07 & 8,2 \\
\hline Divorciado & 17 & 20,0 \\
\hline Casado & 47 & 55,3 \\
\hline Viúvo & 05 & 5,9 \\
\hline
\end{tabular}

Fonte: Dados da pesquisa (2020).

Os sintomas do climatério mais citados nessa pesquisa coincidem com a literatura e chama atenção o fato de que 95,3\% ( $\mathrm{n}=81)$ das mulheres relataram apresentar ondas de calor. A Tabela 2 mostra os sintomas mais comuns no climatério e a frequência deles entre as participantes. 
Tabela 2 - Sintomas climatério relatados.

\begin{tabular}{|c|c|c|}
\hline Item & Freq. & $\%$ \\
\hline \multicolumn{3}{|l|}{ Ressecamento vaginal } \\
\hline Sim & 42 & 49,4 \\
\hline Não & 43 & 50,6 \\
\hline \multicolumn{3}{|l|}{ Diminuição do desejo sexual } \\
\hline Sim & 37 & 43,5 \\
\hline Não & 48 & 56,5 \\
\hline \multicolumn{3}{|l|}{ Ondas de calor } \\
\hline Sim & 81 & 95,3 \\
\hline Não & 04 & 4,7 \\
\hline \multicolumn{3}{|l|}{ Insônia } \\
\hline Sim & 42 & 49,4 \\
\hline Não & 43 & 50,6 \\
\hline \multicolumn{3}{|l|}{ Irritabilidade } \\
\hline Sim & 49 & 57,6 \\
\hline Não & 36 & 42,4 \\
\hline \multicolumn{3}{|l|}{ Ansiedade } \\
\hline Sim & 43 & 50,6 \\
\hline Não & 42 & 49,4 \\
\hline \multicolumn{3}{|l|}{ Depressão } \\
\hline Sim & 20 & 23,5 \\
\hline Não & 65 & 76,5 \\
\hline \multicolumn{3}{|l|}{ Fadiga } \\
\hline Sim & 25 & 29,4 \\
\hline Não & 60 & 70,6 \\
\hline \multicolumn{3}{|l|}{ Dor nos ossos } \\
\hline Sim & 03 & 3,5 \\
\hline Não & 82 & 96,5 \\
\hline \multicolumn{3}{|l|}{ Ressecamento da pele } \\
\hline Sim & 01 & 1,2 \\
\hline Não & 84 & 98,8 \\
\hline \multicolumn{3}{|l|}{ Dor de cabeça } \\
\hline Sim & 12 & 14,1 \\
\hline Não & 73 & 85,9 \\
\hline \multicolumn{3}{|l|}{ Mal estar } \\
\hline Sim & 09 & 10,6 \\
\hline Não & 76 & 89,4 \\
\hline \multicolumn{3}{|l|}{ Ganho de peso } \\
\hline Sim & 03 & 3,5 \\
\hline Não & 82 & 96,5 \\
\hline \multicolumn{3}{|l|}{ Angustia } \\
\hline Sim & 02 & 2,4 \\
\hline Não & 83 & 97,6 \\
\hline \multicolumn{3}{|l|}{ Queda de cabelo } \\
\hline Sim & 01 & 1,2 \\
\hline Não & 84 & 98,8 \\
\hline \multicolumn{3}{|l|}{ Tontura } \\
\hline Sim & 03 & 3,5 \\
\hline Não & 82 & 96,5 \\
\hline
\end{tabular}

Fonte: Dados da pesquisa (2020). 
Os sintomas que aparecem na tabela acima faziam parte do instrumento de coleta e eram apresentados as participantes durante a aplicação do questionário, entretanto durante a pesquisa outros sintomas que não estavam no questionário foram mencionados por algumas participantes que os consideravam também decorrentes do climatério, foram eles: dor nos ossos, ganho de peso, ressecamento da pele, angústia, dor de cabeça, queda de cabelo, mal-estar, tontura. A prevalência de frequiência de todos os sintomas mencionados na pesquisa aparece na Tabela 3.

Tabela 3 - Prevalência (\%) de sintomas do climatério e menopausa.

\begin{tabular}{|c|c|c|}
\hline Item & Freq. & $\%$ \\
\hline Ondas de calor & 81 & 95,3 \\
\hline Irritabilidade & 49 & 57,6 \\
\hline Ansiedade & 43 & 50,6 \\
\hline Ressecamento vaginal & 42 & 49,4 \\
\hline Insônia & 42 & 49,4 \\
\hline Diminuição do desejo sexual & 37 & 43,5 \\
\hline Fadiga & 25 & 29,4 \\
\hline Depressão & 20 & 23,5 \\
\hline Dor de cabeça & 12 & 14,1 \\
\hline Mal estar & 09 & 10,6 \\
\hline Dor nos ossos & 03 & 3,5 \\
\hline Ganho de peso & 03 & 3,5 \\
\hline Tontura & 03 & 3,5 \\
\hline Angustia & 02 & 2,4 \\
\hline Ressecamento da pele & 01 & 1,2 \\
\hline Queda de cabelo & 01 & 1,2 \\
\hline
\end{tabular}

Fonte: Dados coletados durante a pesquisa (2020).

Sobre o uso ou não de alguma substância para tratar os sintomas do climatério 41,1\% (n=35) mulheres informaram que usam algum tipo de planta medicinal, fitoterápicos, hormônios ou outro medicamento alopático para tratamento de um ou vários sintomas. O resultado do teste binomial mostra significância estatística em relação à freqüência de participantes que não usam plantas e fitoterápicos combinados ou não para tratar sintomas da menopausa conforme aparece na Tabela 4.

Tabela 4 - Frequência simples e porcentagem do uso de plantas e fitoterápicos para menopausa.

\begin{tabular}{|c|c|c|c|c|c|}
\hline \multirow{2}{*}{ Variáveis } & \multicolumn{2}{|c|}{ Sim } & \multicolumn{2}{|c|}{ Não } & \multirow{2}{*}{ p-valor } \\
\hline & Freq. & $\%$ & Freq. & $\%$ & \\
\hline Uso de plantas medicinais para menopausa & 21 & 24,7 & 64 & 75,3 & $<0,001^{*}$ \\
\hline Usa fitoterápicos para menopausa & 11 & 12,9 & 74 & 87,1 & $<0,001 *$ \\
\hline Usa plantas e fitoterápicos para menopausa & 03 & 3,5 & 82 & 96,5 & $<0,001 *$ \\
\hline
\end{tabular}

*Diferença estatística ( $\mathrm{p}<0,05$ - Teste binomial). Fonte: Autores.

Já outras mulheres citaram o uso de hormônios ou outros medicamentos alopáticos, em alguns casos, como mostra a Tabela 5, combinado à plantas ou fitoterápicos, para tratar sintomas do climatério e menopausa. Entretanto, quando submetidos à análise através de teste binomial pode-se perceber uma significância estatística acima de $80 \%$ de mulheres que não fazem uso de nenhum tipo de medicação seja natural ou alopática para tratamento dos sintomas. 
Tabela 5 - Frequência simples e porcentagem do uso de hormônios e associação com uso de plantas medicinais ou fitoterápicos.

\begin{tabular}{|c|c|c|c|c|c|}
\hline \multirow{2}{*}{ Variáveis } & \multicolumn{2}{|c|}{ Sim } & \multicolumn{2}{|c|}{ Não } & \multirow{2}{*}{ p-valor } \\
\hline & Freq. & $\%$ & Freq. & $\%$ & \\
\hline Usa hormônio alopático & 16 & 18,8 & 69 & 81,2 & $<0,001 *$ \\
\hline Usa hormônio associado à planta ou fitoterápico & 06 & 7,1 & 79 & 92,9 & $<0,001^{*}$ \\
\hline
\end{tabular}

*Diferença estatística ( $\mathrm{p}<0,05$ - Teste binomial). *Diferença estatística ( $\mathrm{p}<0,05$ - Teste binomial). Fonte: Autores.

Entre as demais participantes $58,9 \%$ (correto $n=50$ ) informaram não usar nada. Dessas 71,4\% ( $\mathrm{n}=42$ ) disseram que embora sentissem algum tipo de incomodo, que em algumas eram bem intensos e em outras moderados, elas acreditavam que isso faz parte de uma fase natural da vida, citaram mães, parentes e amigas como exemplos que também passaram por essa fase e superaram sem ajuda médica, e por isso era apenas uma questão de paciência e resignação, que um dia passaria. 7,1\% (n=2) informaram usar apenas terapias corporais como atividade física e pilates e disseram perceber melhoras. E 21,5\% $(\mathrm{n}=6)$ relataram que já procuraram tratamento médico para auxílio dos sintomas, mas o profissional não prescreveu e que têm medo de tomar algum medicamento por conta própria.

Figura 1 - Distribuição $(\%)$ dos pacientes $(n=85)$ que pediram e não receberam prescrição médica para controle dos sintomas do climatério.

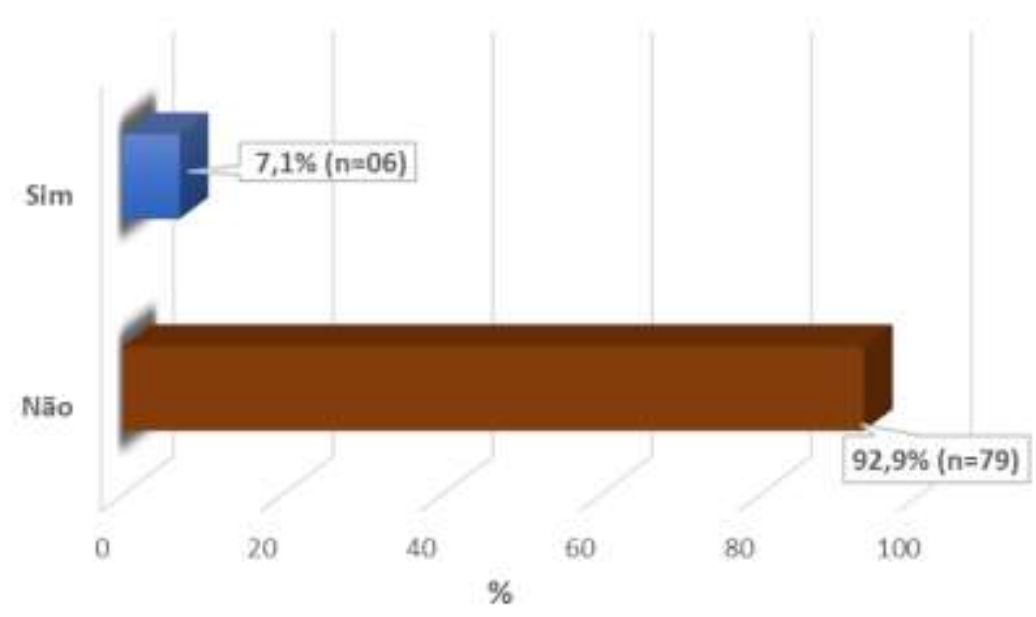

Fonte: Dados coletados durante a pesquisa (2020).

A relação de plantas e fitoterápicos citados nessa pesquisa é apresentada na tabela 6 , e destaca-se aqui o fato de que embora o sintoma mais mencionado pelas participantes tenha sido as ondas de calor, a maioria das participantes disse usar plantas, preparadas como chás, para insônia ou como calmante, mesmo quando a indicação na literatura é para outras sintomatologias. 
Tabela 6 - Plantas medicinais e fitoterápicos utilizados pelas participantes da pesquisa.

\begin{tabular}{cccc}
\hline Item & Freq.
\end{tabular}

\section{Actaea racemosa}

Sim

Não

\section{Camomila}

$\operatorname{Sim}$

Não

\section{Trevo vermelho}

Sim

Não

Erva doce

Sim

Não

02

83

\section{Capim santo}

Sim

07

Não

78

Canela

Sim

Não

Primvit

Sim

Não

\section{Hortelã}

$\operatorname{Sim}$

Não

Folha de laranja

Sim

Não

\section{Isoflavona}

Sim

Não

\section{Miconia alvicans}

Sim

2,4

97,6

97,6

97,6

98,8

84

01

1,2

84

98,8

02

2,4

83

97,6

Não
01

84

01

84

1,2

98,8

03

3,5

82

96,5

1,2

98,8 


\section{Alecrim}

$\operatorname{Sim}$

01

1,2

Não

\section{Cidreira}

Sim

04

Não

Amora

Sim

Não

\section{Citocalmy}

Sim

Fonte: Dados coletados durante a pesquisa (2020).

Entre as plantas que aparecem na Tabela 7 a Canela (Cinnamomum zeylanicum) citada 1,2\% (n=1) não teve nenhuma indicação calmante, sedativa ou para qualquer outra sintomatologia comum ao climatério e menopausa na literatura, tendo apenas referências a suas propriedades culinárias, antiinflamatórias, antifúngicas, antioxidante. Situação semelhante à Canela de velho (Miconia alvicans) mencionada por 1,2\% ( $\mathrm{n}=1)$ de participante e que não apresenta relação com sintomas do climatério e menopausa na literatura, sendo indicada para casos de dores reumáticas e musculares.

As demais plantas citadas nessa pesquisa apresentam respaldo científico em menor ou maior grau para sintomatologias citadas pelas participantes, como por exemplo, a cidreira e o alecrim referenciados em alguns estudos como ansiolíticos leves. E a Actaea racemosa 2,4\% ( $n=2)$ e o Trevo vermelho 2,4\% ( $n=2)$ que apresentam vários estudos relacionando-as a efeitos benéficos para alívio dos sintomas do climatério.

Com relação especificamente aos fitoterápicos embora um número menor de mulheres tenha referido uso, de forma individual ou combinada, todos os fitoterápicos citados nessa pesquisa tem respaldo na literatura para tratamento de sintomatologia do climatério. 
Research, Society and Development, v. 10, n. 10, e206101018752, 2021

(CC BY 4.0) | ISSN 2525-3409 | DOI: http://dx.doi.org/10.33448/rsd-v10i10.18752

Tabela 7 - Ordem decrescente de consumo de Plantas e Fitoterápicos (Prevalência \%).

\begin{tabular}{|c|c|c|}
\hline Item & Freq. & $\%$ \\
\hline Camomila & 13 & 15,3 \\
\hline Capim santo & 07 & 8,2 \\
\hline Cidreira & 04 & 4,7 \\
\hline Amora & 04 & 4,7 \\
\hline Isoflavona & 03 & 3,5 \\
\hline Citocalmy & 03 & 3,5 \\
\hline Actaea racemosa & 02 & 2,4 \\
\hline Trevo vermelho & 02 & 2,4 \\
\hline Erva doce & 02 & 2,4 \\
\hline Hortelã & 02 & 2,4 \\
\hline Canela & 01 & 1,2 \\
\hline Primavit & 01 & 1,2 \\
\hline Folha de laranja & 01 & 1,2 \\
\hline Miconia alvicans & 01 & 1,2 \\
\hline Alecrim & 01 & 1,2 \\
\hline
\end{tabular}

Fonte: Dados coletados durante a pesquisa (2020).

Das 24 mulheres que informaram fazer uso de plantas medicinais 45,8\% (n=11) disseram cultivar em casa ou adquirir de vizinhas ou amigas que cultivam, e 54,2\% ( $\mathrm{n}=13$ ) disseram comprar em lojas de produtos naturais ou supermercados. Das 14 que usam fitoterápicos 11 compram em farmácias tradicionais e 3 em farmácias de manipulação. Sobre quem fez a indicação e orientou sobre o uso, o grupo que consome plantas medicinais disseram que foram aconselhadas a consumir por amigas, mães e outros familiares. Com relação ao grupo que usa fitoterápicos apenas 1 disse ter sido orientada por uma amiga, as outras 13 receberam prescrição médica.

No tocante a posologia e frequiência de consumo pôde-se perceber uma irregularidade com relação a horários e padronização das dosagens. A maioria consome plantas medicinais através de chás, ensinados por familiares ou amigos e feitos de maneira aleatória, sem uma medida exata. Geralmente o consumo se dá apenas uma vez ao dia e em alguns casos como mostra a tabela 8 esse consumo acontece semanalmente. 
Tabela 8 - Valores de frequência simples e porcentagem sobre quantidade e freqüência de consumo de plantas medicinais e fitoterápicos.

\begin{tabular}{|c|c|c|}
\hline Item & Freq. & $\%$ \\
\hline \multicolumn{3}{|l|}{ Frequência de xícaras } \\
\hline Diariamente & 16 & 69,6 \\
\hline Dias alternados & 04 & 17,4 \\
\hline Semanalmente & 03 & 13,0 \\
\hline \multicolumn{3}{|l|}{ Quantidade de xícaras } \\
\hline 01 & 18 & 78,3 \\
\hline 02 & 04 & 17,4 \\
\hline 03 & 01 & 4,3 \\
\hline \multicolumn{3}{|l|}{ Frequência de cápsulas } \\
\hline Diariamente & 08 & 88,9 \\
\hline Dias alternados & 01 & 11,1 \\
\hline \multicolumn{3}{|l|}{ Quantidade de cápsulas } \\
\hline 01 & 07 & 77,8 \\
\hline 02 & 01 & 11,1 \\
\hline 03 & 01 & 11,1 \\
\hline \multicolumn{3}{|l|}{ Percebe melhoras } \\
\hline Sim & 27 & 90,0 \\
\hline Não & 03 & 10,0 \\
\hline
\end{tabular}

Fonte: Dados coletados durante a pesquisa (2020).

Essa irregularidade no consumo e dosagem pode comprometer o resultado esperado e estar relacionado às respostas daquelas que informaram não perceber melhoras nos sintomas.

Já os fitoterápicos, embora consumidos por menor número de participantes, teve um consumo mais regular em relação à posologia e freqüência, isso pode estar relacionado ao fato de que apenas uma participante da pesquisa informou usar fitoterápicos por recomendação de uma amiga, as demais usam sob orientação e prescrição médica.

Sobre outras doenças que acometem as participantes dessa pesquisa os transtornos depressivos e de ansiedade foram os mais citados, seguido de hipertensão, chama atenção aqui o fato de apenas 1 participante mencionar diagnóstico de osteoporose, doença que segundo a literatura aumenta sua prevalência em mulheres na menopausa. Quanto ao uso de outras medicações alopáticas para doenças além da sintomatologia clássica da menopausa, percebe-se um consumo expressivo de ansiolíticos e antidepressivos, que aparece em 17 questionários, seguido de hipertensivos com relato de consumo por 9 participantes.

\section{Discussão}

Embora muitas mulheres relatem conviver com sintomas do climatério, evidencia-se que poucas têm conhecimento sobre fitoterápicos e plantas medicinais para essa finalidade. A maioria das participantes desse estudo faz uso de plantas na forma de chá para insônia e ansiedade sem saber a relação desses sintomas com o climatério. Estudo realizado por Ghizi e Mezzomo (2015) sobre o consumo de plantas medicinais por moradores de Curitiba para tratamento de patologias variadas, mostrou a utilização de chás para efeitos calmantes como o segundo mais citado. Pesquisas realizadas por Paiva et al (2017); Schiavo et al (2017); Silva, Rocha e Pereira (2020), mostram que a prevalência do consumo de plantas medicinais através de chás é um dado comum em estudos dessa área. 
Um estudo realizado por Silva, Marini e Melo (2015) na Paraíba mostrou que a preferência do consumo de plantas medicinais na forma de chá se dá pela facilidade, praticidade, rapidez do preparo e eficácia no alívio dos sintomas, quando comparada a outras preparações como garrafadas, lambedores, banhos, entre outros, que às vezes precisam de combinações de ervas e alguns cuidados nas técnicas de preparo. Vale lembrar ainda que a forma de preparo e utilização das plantas medicinais é de grande relevância para os resultados do tratamento, pois pode interferir na ação terapêutica.

Explicita-se que o conhecimento sobre o uso de plantas medicinais acontece principalmente através da transmissão oral de saberes populares entre gerações, o que corrobora com outros estudos como o de Schiavo et al (2017) e de Silva, Rocha e Pereira (2020), que mostram que o conhecimento a cerca do uso de plantas medicinais se dá através de familiares ou pessoas do meio social ao qual se está inserido, sendo comumente transferido através de gerações.

O uso de plantas medicinais para fins terapêuticos no país segundo estudo de Caetano et al (2015) caracteriza-se por experiências empíricas e pouca comprovação científica, o que pode levar à utilização errônea, uma vez que pode haver imprecisão nas espécies botânicas, além de erros na maneira de cultivo, armazenamento e preparo adequado das formulações caseiras.

Nesse estudo percebeu-se que algumas mulheres que fazem uso de chás não têm regularidade no consumo, utilizando apenas quando apresentam sintomas mais acentuados, e a maioria, mesmo das que consomem diariamente, utiliza apenas uma xícara por dia, sem nenhum embasamento técnico ou científico da posologia adequada para cada espécie vegetal. Essa prática irregular e empírica pode ser o cerne das respostas daquelas que disseram não perceber melhoras nos sintomas e justificada pela baixa indicação por parte dos profissionais de saúde, conforme relato das participantes da UBS.

Outro achado que parece estar relacionado ao consumo empírico de plantas para fins terapêuticos é a baixa escolaridade da maioria das participantes e o baixo poder aquisitivo delas. Desde o final da década de 70, a OMS já reconhecia que cerca de $80 \%$ da população de países em desenvolvimento usam plantas medicinais nos cuidados básicos em saúde, e que essa prática está relacionada às condições socioeconômicas e culturais (Brasil, 2008b; Beleza, 2016).

Geralmente a utilização das plantas medicinais é feita por pessoas de menor poder aquisitivo, muitas vezes por falta de recursos financeiros para aquisição de medicamentos alopáticos, dificuldade de acesso, pela vasta biodiversidade brasileira de plantas, pela crença de que por ser um produto natural é inofensivo, ou simplesmente pela tradição cultural. A baixa escolaridade e o baixo poder aquisitivo da maioria das participantes dessa pesquisa, reforça essa assertiva e corrobora com outros estudos como o de Cruz et al (2017); Schiavo et al (2017), que mostram que as plantas medicinais estão entre as principais formas de tratamento de doenças, nas regiões economicamente mais pobres do país.

Com relação aos meios de aquisição das plantas medicinais o cultivo em domicílio e a compra em lojas de produtos naturais e supermercados foram citados em percentual aproximado, dado esse que difere de alguns estudos como o de Cruz et al (2017), Silva; Rocha e Pereira (2020), que apontam o cultivo em domicílio como a principal forma de obtenção desse produto.

Nesse estudo o consumo de fitoterápicos ficou bem aquém do consumo de plantas, e chamou atenção o fato da maioria das participantes que informaram usar fitoterápicos serem atendidas no ambulatório da FACS e terem o uso destes respaldado por orientação médica. Situação bem diferente da observada entre as participantes da UBS, onde apenas 1 mulher usa fitoterápicos para sintomas de climatério e menopausa, e disse que foi orientada por uma amiga. Estudo conduzido por Gelatti, Oliveira e Colet (2016) mostra semelhanças a esse, quanto ao maior consumo de plantas comparado ao de fitoterápicos, e em relação a baixa prescrição de ambos por profissionais da Estratégia Saúde da Família que atuam nas UBS.

Para essas autoras um dos possíveis motivos para o baixo consumo de fitoterápicos quando comparados as plantas medicinais pode ser o receio de que esses produtos apresentem maior risco de efeitos adversos, uma vez que passam por manipulação farmacêutica, a facilidade do cultivo de plantas, e o custo mais elevado dos fitoterápicos. 
O valor de medicamentos fitoterápicos, que atualmente chega a ser muitas vezes mais caros do que algumas medicações alopáticas, bem como a dificuldade de acesso a esses produtos por moradores de localidades longe dos grandes centros urbanos também contribuem para a pouca popularidade desse produto entre a população de menor poder aquisitivo, e residentes em cidades de pequeno porte ou zonas rurais.

Estudo realizado por Gelatti; Oliveira e Colet (2016) mostra que embora os fitoterápicos tenham segurança e eficácia comprovada por evidências clínicas, em contraposição as plantas medicinais que muitas vezes são usadas na forma de preparações caseiras não padronizadas, esses ainda não tem seu uso popularizado. Seja por descrença de que por passar por intervenção farmacêutica são menos naturais, pela facilidade de cultivo, acesso e baixo custo em relação às plantas.

Estudos como o de Gelatti; Oliveira e Colet (2016) mostram também que a prescrição de fitoterápicos e plantas medicinais na Atenção Básica de Saúde é mais comum em municípios que desenvolvem o Programa de Farmácias Vivas. Criado em 2010 esse programa regulamenta as boas práticas de processamento e armazenamento de plantas medicinais, preparação e dispensação de produtos magistrais no Sistema Único de Saúde (SUS), e busca promover do uso racional das plantas medicinais na atenção básica, através da união entre o resgate do conhecimento popular e os conhecimentos científicos (Brasil, 2010).

O consumo de fitoterápicos e plantas medicinais sem a devida orientação profissional pode trazer danos à saúde dessas mulheres. Apesar da crença de que esses produtos são naturais e por isso não oferecem risco, e embora muitos desses produtos apresentem menores efeitos colaterais quando comparados a medicações alopáticas, substâncias químicas presentes em algumas plantas podem apresentar toxicidade ao organismo (Souza et al, 2019). Leal e Tellis (2015) alertam para os riscos da ingestão de plantas medicinais e fitoterápicos sem o devido conhecimento sobre a segurança dos mesmos, e principalmente o risco de interação quando consumido junto a medicações alopáticas.

Nessa pesquisa foi possível constatar que 45\% ( $\mathrm{n}=38)$ das participantes usam medicações alopáticas para tratamento de doenças como depressão, ansiedade, hipertensão, diabetes, osteoporose e tireóide. Desse total 23,7\% (n=9) mulheres fazem uso dessa medicação associada ao consumo de plantas ou fitoterápicos sem conhecimento das possíveis interações adversas entre as substâncias.

Em relação à vivência do climatério e menopausa o consumo esporádico de uma ou outra planta ou algum remédio natural, como elas se referiram aos fitoterápicos, associado à medicação de uso contínuo parece não preocupar. Na visão das participantes, essa é uma fase que toda mulher cedo ou tarde vai vivenciar, uma fase natural da vida da qual a maioria das participantes não associa a processos patológicos e por isso não vê relação de uma possível interação entre tratamento de sintomas do climatério e menopausa com o de outra patologia pré-existente.

Perceber o climatério e a menopausa como um período natural da vida coincide com a definição da OMS, que afirma ser essa fase um processo biológico natural do envelhecimento feminino e não uma patologia (Brasil, 2008a). Entretanto, durante a pesquisa evidenciou-se que essa visão acerca do climatério é embasada não pelo conhecimento científico, mas pelas experiências empíricas vivenciadas no cotidiano familiar.

Chamou a atenção o fato de 44,7\% ( $n=38)$ participantes alegarem que apesar de sentirem muitos incômodos decorrentes do climatério, dentre eles os conhecidos "calorões", procuram suportar as adversidades desse período sem buscar alívio para esses sintomas. Pois para elas foi assim também com suas mães, irmãs e outras mulheres da família e todas superaram sem qualquer tipo de intervenção, era apenas uma questão de esperar pacientemente o tempo passar que um dia os incômodos acabariam.

Estudos como o de Zanette et al (2011) mostram que os "calorões", é um dos sintomas clássicos do climatério que afeta $85 \%$ das mulheres que vivencia essa fase e que afetam consideravelmente a qualidade de vida. Já Lomônaco, Tomaz e Ramos (2015) afirmam que a maioria das mulheres tem pouca informação sobre climatério e menopausa, o que levam - nas a 
encarar essa fase como um ritual de passagem para a velhice e, portanto, uma fase natural da vida que todas terão que atravessar.

Esse comportamento remete a pensar que as questões sociais e culturais influenciam nas vivências do climatério: não falar da fase pela vergonha em expor algo íntimo, não poder parar as atividades profissionais e cotidianas para tratar os sintomas, as questões de ansiedade e depressão, a baixa autoestima relacionada ao fim da idade reprodutiva.

No caso específico dessa pesquisa o baixo nível de escolaridade e renda da maioria das participantes muitas vezes suscita situações de vulnerabilidade que levam a depressão, ansiedade, conflitos familiares e conjugais, que vão se confundir com sintomas psicossociais desse período fisiológico, chegando algumas vezes a afetar consideravelmente a qualidade de vida.

Esses achados corrobora com os trabalhos de Lomônaco, Tomaz e Ramos (2015), Serpa et al (2016) que mostram que além das alterações endócrinas e fisiológicas comuns ao climatério e menopausa esse período coincide também com algumas mudanças que ocorrem a nível social e psicológico. Acontecimentos como aposentadoria, saída dos filhos da casa materna, divórcio, viuvez, conflitos familiares em geral, afetam diretamente a percepção do papel social da mulher tanto na família como na sociedade e que podem ser vivenciados de maneira mais intensa ainda por mulheres com menor nível de escolaridade e poder aquisitivo.

Sintomas como ondas de calor, ressecamento vaginal, diminuição do desejo sexual e irritabilidade que foram os mais citados nessa pesquisa podem interferir significativamente na vida sexual das mulheres e nos relacionamentos. Portanto, a vivência do climatério e menopausa é um fenômeno social e compartilhado. Considerando que 63,5\% (n=54) das participantes informaram ter parceiros fixos, a vivência dessa fase pode interferir na visão da feminilidade e consequentemente na vida sexual e afetiva dessas mulheres.

Para as participantes dessa pesquisa essa situação é intensificada pelas condições socioeconômicas, uma vez que a escassez de recursos limita o acesso a serviços de saúde, tratamentos hormonais e terapias integrativas, medicamentos e cosméticos que pudessem mascarar o retardar os sinais de envelhecimento. Estudo realizado por Miranda; Ferreira e Corrente (2014) com mulheres climatéricas atendidas em UBS, mostrou que as atitudes e percepções das mulheres com relação a menopausa tem relação com a intensidade dos sintomas e efeitos do climatério em todas as suas dimensões, biológica, psicológica, social e emocional.

A percepção e vivência do climatério e menopausa é uma experiência individual, influenciada por diferentes dimensões da vida. Nesse estudo ficou claro que a maioria das participantes, desconhecem ou tem poucas informações sobre tratamento para alívio dos sintomas, principalmente de terapias fora da reposição hormonal.

Embora a medicina e a indústria farmacêutica desde a década de 60 tenham popularizado a terapia de reposição hormonal e apontado a possibilidade de se viver o climatério com qualidade de vida e livre de alguns sinais de envelhecimento evidenciados nesse período, a medicalização dessa fase da vida, limita-se as mulheres favorecidas econômica e socialmente (Franciscis et al., 2019; Wender; Pompei; Fernandes, 2014). Pois a maioria das mulheres, principalmente as de menor renda, que corresponde ao público principal atendido no serviço público, não tem acesso a esse tratamento, além de nem sempre terem indicação para seu uso e o medo de efeitos colaterais.

Por outro lado, nos últimos anos tem crescido o interesse de pesquisadores e profissionais de saúde em estudos sobre fitoterapia e plantas medicinais para tratamento de doenças, inclusive para sintomas do climatério. Entretanto, apesar de ser uma prática antiga, que vem despertando crescente interesse de uma parcela da comunidade científica nos últimos anos, o consumo de plantas medicinais e fitoterápicos ainda enfrenta grande resistência, principalmente por parte de alguns profissionais de saúde, pois ainda é pouco valorizada cientificamente.

Mesmo diante da crescente cientificidade envolvendo a temática, a indústria farmacêutica ainda coloca a medicina alopática como principal protagonista da área da saúde e consequentemente influencia a conduta de médicos e outros 
profissionais da área no momento da prescrição. Para Antonio, Tesser e Moretti-Pires (2013, p.628) “(...) a formação dos profissionais em saúde fundamenta-se no saber técnico-científico, vinculada, no geral, à perspectiva de conhecimentoregulação, unidirecional e prescritivo", portanto fortemente influenciada pela ideologia do saber biomédico, que se mostra pouco aberta a mudanças e as discussões que envolvem a validade do saber popular e tradicional sobre plantas medicinais.

Em relação a esse assunto o Brasil tem uma situação peculiar quando comparado a outros países, pois possui uma imensa variedade de plantas nativas. Dados do Projeto Flora do Brasil (2016) o país possuía mais de 46 mil espécies de plantas, e muito pouco se é aproveitado pela indústria farmacêutica para fins terapêuticos. De acordo com Antonio, Tesser e Moretti-Pires (2013) as exigências para se garantir qualidade, eficácia e segurança, impostas pela legislação brasileira dificulta a produção e a popularização do uso de fitoterápicos, pois são poucos os produtores locais que tem condições de atender todas as exigências legais.

Toda essa situação se reflete nessa pesquisa através das falas das participantes atendidas na UBS quando informam que o pouco conhecimento que tem de plantas medicinais para sintomas do climatério procede das relações sociais e familiares. Em relação aos fitoterápicos, o único registro nesse grupo também aconteceu por recomendação de amiga. Esse fato evidência a pouca, ou ausente, aproximação da temática por parte dos profissionais da atenção básica e reflete o quanto os programas de atenção à saúde da mulher ainda restringe suas ações a mulheres na fase reprodutiva.

Por outro lado, chamou atenção a situação inversa vivenciada pelas mulheres atendidas no ambulatório da FACS. Nesse grupo o consumo de plantas medicinais foi menor comparado ao das mulheres atendidas na UBS, mas todas as prescrições de fitoterápicos registradas nesse estudo foram realizadas por estudantes e professores do curso de medicina. Esse fato provoca a reflexão sobre um possível aumento do conhecimento e aceitação dessas práticas nos cursos da área de saúde, que pode estar relacionado ao crescente interesse que a temática vem despertando nos últimos anos.

\section{Conclusão}

O presente estudo mostrou que as mulheres têm pouca informação sobre tratamentos para sintomas de climatério e menopausa, que prevalece ainda muito receio sobre os efeitos da terapia de reposição hormonal e quase nenhum conhecimento sobre outras terapias complementares adequadas a esse período. No tocante ao uso de plantas medicinais e fitoterápicos para esse fim percebeu-se um predomínio de consumo de plantas em relação aos fitoterápicos, entretanto, os primeiros são usados a partir de conhecimento adquiridos por tradição.

De acordo com os resultados da pesquisa as mulheres não utilizam as plantas medicinais e fitoterápicos como estratégias de baixo custo e de baixo efeito colateral para tratamento dos sintomas do climatério.

Embora o sintoma mais apontado como incomodo, e tradicionalmente mais identificado com essa fase da vida tenha sido as ondas de calor, a maior parte do consumo de plantas medicinais se dá através de chás para tratar insônia e ansiedade, a maiorias das vezes sem associação ao climatério ou menopausa. Já os fitoterápicos tem pouco consumo, sendo a isoflavona o componente mais comum deles.

Nesse trabalho ficou evidente a ausência de ações voltadas para mulheres no climatério por parte dos profissionais da atenção básica, em contraposição a essa prática na atenção especializada oferecida por instituição de ensino. Como também foi visível o distanciamento dos profissionais de saúde da UBS em relação às informações sobre plantas medicinais e fitoterápicos. Mostrando a necessidade de mais capacitação e treinamentos sobre essa temática para esses profissionais.

Todos os fitoterápicos citados nos resultados dessa pesquisa têm indicação para tratamento de um ou mais sintomas do climatério conforme legislação vigente no país. Já as plantas medicinais mencionadas pelas participantes nem sempre tinham indicações correspondentes ao uso referido por elas. 
Apesar do uso de plantas medicinais ser uma prática popular no Brasil, nesse estudo foi possível perceber que o número de publicações nessa área ainda deixa a desejar, principalmente no que se refere à qualidade metodológica dos estudos. Outra limitação encontrada diz respeito ao nome popular de algumas plantas que varia de acordo com algumas regiões do país, como é o caso do capim santo e da cidreira que às vezes se confunde.

Espera-se que esse trabalho possa contribuir de alguma forma para outros estudos tanto no que se refere à área de plantas medicinais e fitoterápicos, como também sobre climatério e menopausa, visto que essas temáticas ainda apresentam muitas possibilidades de estudo. Como por exemplo, mais estudos experimentais utilizando algumas das plantas ou substâncias citadas na literatura como efetivas para amenizar sintomas climatéricos, bem como, estudos que avaliem as ações e políticas de saúde voltadas para mulheres nessa fase da vida.

\section{Referências}

Aidelsburger, P., Schauer ,S., Grabein, K., \& Wasem, J. (2012). Alternative methods for the treatment of post-menopausal troubles. African Journal Traditional Complementary and Alternative Medicine. http://journals.lww.com/menopausejournal/Abstract/2012/02000/Yoga_decreas es_insomnia_in _postmenopausal_women__.13.aspx.

Albuquerque, G. P. M., Abrão, F. M. S., Almeida, A. M., Alves, D. L. R., Andrade P. O. N., \& Costa, A. M. (2019) Qualidade de vida no climatério de enfermeiras atuantes na atenção primária. Rev Bras Enferm. 72(3), 161-168. http://dx.doi.org/10.1590/0034-7167-2018-0306.

Antonio, G. D., Tesser, C. D., \& Moretti-Pires, R. O. (2013). Contribuições das plantas medicinais para o cuidado e a promoção da saúde na atenção primária. Interface: comunicação, saúde e educação. 17(46), 615 - 33.

Barra, A. A. L., Albergaria, D. A., Mariano, F. M., Dantas, J. B., Pinto, K. M. C., \& Resende, N. M. (2014). Terapias alternativas no climatério. FEMINA. 42 (1), 27-31.

Beleza, J. A. M (2016). Plantas medicinais e fitoterápicos na atenção primária à saúde: contribuição para profissionais prescritores. Monografia [Especialização em Gestão da Inovação de Medicamentos da Biodiversidade ]. Instituto de Tecnologia em Fármacos.

Brasil (2008a). Manual de Atenção à Mulher no Climatério/Menopausa. http://bvsms.saude.gov.br/bvs/publicacoes/manual_atencao_mulher_climaterio.pdf.

Brasil (2008b). Política Nacional de Práticas Integrativas e Complementares no SUS: PNPIC: atitude de ampliação de acesso. https://bvsms.saude.gov.br/bvs/publicacoes/politica_nacional_praticas_integrativas_complementares_2ed.pdf.

Brasil (2009). Programa Nacional de Plantas $\quad$ Medicinais $\quad$ Fitoterápicos. https://bvsms.saude.gov.br/bvs/publicacoes/programa_nacional_plantas_medicinais_fitoterapicos.pdf.

Brasil (2010). Portaria $N^{o}$ 886, de 20 de abril de 2010. Institui a Farmácia Viva no âmbito do Sistema Único de Saúde (SUS). https://bvsms.saude.gov.br/bvs/saudelegis/gm/2010/prt0886_20_04_2010.html.

Bueno, M. J. A, (2016). Manual de plantas medicinais e fitoterápicos utilizados na cicatrização de feridas. Dissertação [Mestrado Profissional em Ciências Aplicadas à Saúde]. Univás. https://www.univas.edu.br/Egressos_Web/26.pdf.

Caetano, N. L. B., Ferreira, T. F., Reis, M. R. O., Neo, G. G. A., \& Carvalho, A. A. (2015). Plantas medicinais utilizadas pela populacao do municipio de Lagarto- SE, Brasil - enfase em pacientes oncologicos. Rev. Brás. de plantas med. 17(4), 748 -756.

Cardoso, E. C., \& Camargo, M. J. G. (2017). Terapia Ocupacional em Saúde da Mulher: impacto dos sintomas do climatério na atividade profissional. Tempus, actas de saúde colet, 11(1), 153-167.

Cruz, V. M. S., Gonçalves, A. L., Campos, J. R P., \& Reis, A. R. S. (2017). Aspectos socioeconômicos e o cultivo de plantas medicinais em quintais agroflorestais urbanos (QAF) no município de Breu Branco, Pará, Brasil. Enciclop. Biosfera, 14 (25) 158 - 170.

Fernandes, C., \& Farias, J. M. (2017). Perfil Socioeconômico, Hábitos de Vida de Mulheres no Período de Menopausa. http://repositorio.unesc.net/bitstream/1/4854/1/Camila\%20Fernandes.pdf.

FLORA DO BRASIL (2020). Algas, fungos e plantas. Em construção. Lançamento de plataforma online em 2016. Jardim Botânico do Rio de Janeiro. http://floradobrasil.jbrj.gov.br/reflora/PrincipalUC/PrincipalUC.do.

Franciscis, P., Colacurci, N., Riemma, G., Conte, A., Pittana, E., Guida, M., \& Schiattarella, A. (2019). A Nutraceutical Approach to Menopausal Complaints. Medicina, 55(544), 1-16. 10.3390/ medicina55090544.

Manica, J., Bellaver, E. H., \& Zancanaro, V. (2019). Efeitos das terapias na menopausa: uma revisão narrativa da literatura. J. Health BiolSci, 7(1), 82-88.

Gelatti, G. T., Oliveira, K. R., \& Colet, C. F. ((2016). Potenciais interações relacionadas ao uso de medicamentos, plantas medicinais e fitoterápicos em mulheres no período do climatério. J. res.: fundam. care. Online, 8(2), 4328-4346. 
Ghizi, A., \& Mezzomo, T. R. (2015). Uso de Plantas Medicinais e Satisfação de Consumidores de Lojas de Produtos Naturais do Mercado Municipal de Curitiba, PR. Rev. Fitos, 9(2), 73-159.

Ibiapina, W. V., Leitão, B. P., Batista, M. M., \& Pinto, D. S. (2014). Inserção da Fitoterapia na atenção primária aos usuários do SUS. Rev. Ciência Saúde Nova Esperança, 12(1), 58-68.

Leal, L. R., \& Tellis, C. J. M. (2015). Farmacovigilância de plantas medicinais e fitoterápicos no Brasil: uma breve revisão. Rev. Fitos, 9(4), 253 - 303.

Lomônaco, C., Tomaz, R. A. F., \& de Oliveira Ramos, M. T. (2015). O impacto da menopausa nas relações e nos papéis sociais estabelecidos na família e no trabalho. Reprodução \& Climatério, 30(2), 58-66.

Paiva, K. O., Oliveira, G. L., Farias, D. F. A., \& Müller, T. S. (2017). Plantas medicinais utilizadas em transtornos do sistema geniturinário por mulheres ribeirinhas, Caravelas, Bahia. Rev. Fitos, 1, $92-98$.

Pereira, A. S., Shitsuka, D. M., Parreira, F. J., \& Shitsuka, R. (2018). Metodologia da pesquisa científica. UFSM.

Rocha, B. M. A., Pereira, M. S. V., \& Carneiro, J. Q. (2018). Terapias Complementares: fitoterapia como opção terapêutica no climatério e menopausa. Revista de Ciências da Saúde Nova Esperança, 16 (1), 16-25. http://dx.doi.org/10.17695/issn.2317-7160.v16n1a2018p16-25.

Sampieri, R.H., Collado, C. F., \& Lucio, P. B. (2013). Metodologia de Pesquisa. McGraw-Hill.

Serpa, M. A., Lima, A. A., Guimarães, A. C. P., Carrilo, M. R. G. G., Coura-Vitalb, W., \& Veloso, V. M. (2016). Fatores associados à qualidade de vida em mulheres no climatério. Reprod Clim, 31(2), 76-81.

Schiavo, M., Gelatti, G.T., Oliveira, K. R., Bandeira, V. A. C., \& Colet, C. F. (2017). Conhecimento sobre plantas medicinais por mulheres em processo de envelhecimento. Semina: Ciências Biológicas e da Saúde, 38(1), 45 - 60. http://dx.doi.org/10.5433/1679-0367.2017v38n1p45.

Silva, L. D. C., \& Mamede, M.V. (2020). Prevalência e intensidade de sintomas climatéricos em mulheres com doença arterial coronariana. RevFunCare Online. 12, 305-312. http://www.seer.unirio.br/index.php/cuidadofundamental/article/view/6755/pdf_1.

Silva, M. D. P., Marini, F. S., \& Melo, R. S. (2015). Levantamento de plantas medicinais cultivadas no município de Solânea, agreste paraibano: reconhecimento e valorização do saber tradicional. Rev. Bras. Pl. Med, 17(4), 881-890.

Silva, O. B., Rocha, D. M., \& Pereira, N. V. (2020). O saber tradicional e o uso de plantas medicinais por moradores do assentamento padre Ezequiel em Mirante da Serra - RO, Brasil. Biodiversidade, 19(1), 77 - 96.

Souza, N. R. R, Viana, M. E. L., Miranda, M. L. C., Guimarães, B. C. S., Miranda, M. L., \& Souza, J. H. K. (2019). Relação entre terapia de reposição hormonal no climatério e o desenvolvimento de neoplasias. Braz. J. Surg. Clin. Res, 25 (2), 135-143.

Wender, C. O. M., Pompei, L. M., Fernandes, C. E. (2014) Consenso Brasileiro de Terapêutica Hormonal da Menopausa. Associação Brasileira de Climatério (SOBRAC). Leitura Médica. http://sobrac.org.br/consenso_brasileiro_de_th_da_menopausa_2018.html.

Xavier, P. F. P., \& Trindade, A. P. N. T. (2018). Avaliação do risco de queda e equilíbrio em mulheres no climatério. Revista Kairós-Gerontologia, 21(2), 155-170. https://doi.org/10.23925/2176-901X.2018v21i2p155-170.

Zanette, V. C., Rossato, A. E., Citadini-Zanette, V., \& Bernardi, F. B. C. (2011). Prevalência do uso da fototerapia para alívio de sintomas apresentados em pacientes climatéricas. Arquivos Catarinenses de Medicina. http://www.acm.org.br/revista/pdf/artigos/840.pdf. 